\title{
Effects of Dapagliflozin Adjunct to Insulin on Glycemic Variations in Patients with Newly Diagnosed Type 2 Diabetes: A Randomized, Controlled, Open-Labeled Trial
}

\author{
Lan-lan Jiang, ${ }^{1}$ Peng Zhang, ${ }^{1}$ Bing-li Liu, ${ }^{1}$ Reng-na Yan, ${ }^{1}$ Lei Ye, ${ }^{2}$ Jian-hua Ma $\left(\mathbb{D},{ }^{1}\right.$ \\ and Feng-fei $L i{ }^{1}{ }^{1}$ \\ ${ }^{1}$ Department of Endocrinology, Nanjing First Hospital, Nanjing Medical University, Nanjing, China \\ ${ }^{2}$ National Heart Research Institute Singapore, National Heart Centre Singapore, Singapore \\ Correspondence should be addressed to Jian-hua Ma; majianhua196503@126.com and Feng-fei Li; lifengfei2005@sina.com
}

Received 4 November 2020; Accepted 10 August 2021; Published 29 August 2021

Academic Editor: Yunjuan Gu

Copyright (C) 2021 Lan-lan Jiang et al. This is an open access article distributed under the Creative Commons Attribution License, which permits unrestricted use, distribution, and reproduction in any medium, provided the original work is properly cited.

\begin{abstract}
Background. This study is aimed at investigating whether dapagliflozin adjunct to insulin therapy further improves glycemic control compared to insulin therapy alone in patients with newly diagnosed type 2 diabetes (T2D). Methods. This singlecentre, randomized, controlled, open-labeled trial recruited newly diagnosed T2D patients. Subjects were randomized $1: 1$ to the dapagliflozin add-on to continuous subcutaneous insulin infusion (CSII) group (DAPA) or the CSII therapy group for 5 weeks. Standard meal tests were performed 3 times at days -3, 7, and 35 for glucose, C-peptide, and insulin level determination. Two-time continuous glucose monitoring (CGM) was performed at baseline and at the end of the study. The primary endpoint was the difference in the mean amplitude of glycemic excursions (MAGEs) between the groups. Results. A total of 66 subjects completed the study, with 34 and 32 patients in the DAPA and CSII groups, respectively. Patients in the DAPA group exhibited significant decreases in MAGE levels at the endpoint. We also observed that patients in the DAPA group had a lower homoeostasis model assessment insulin resistance (HOMA-IR) and a higher homoeostasis model assessment B (HOMA-B) value at 1 week and 5 weeks compared to those with insulin therapy, respectively. In addition, our data showed that patients in the DAPA group showed a significantly lower insulin dose $(0.07 \mathrm{U} / \mathrm{kg})$ and weighed less than those in the CSII group. Conclusion. Our data indicate that dapagliflozin adjunct to insulin is a safe and effective therapy for improving glycemic variations, insulin sensitivity, and weight loss in newly diagnosed T2D patients.
\end{abstract}

\section{Introduction}

The incidence and prevalence of type 2 diabetes (T2D) are increasing worldwide partly due to changes in dietary habits and a lack of exercise [1-3]. Insulin therapy is the mainstay of treatment for achieving target hemoglobin Alc $\left(\mathrm{HbA}_{1 \mathrm{c}}\right)$ levels in patients with T2D. However, this treatment is associated with hypoglycemia [4-6] (especially in older male diabetic patients [7]), increased blood pressure [8], and weight gain [9]. Other concerns regarding the use of insulin, such as adherence, preferences, and resource allocation, have also been well documented [10]. Moreover, intensification of insulin is associated with glucagon imbalances and increased rate of gastric emptying, which may lead to a dramatically increased glycemic variations (GV) [11]. As such, an adjunct agent for insulin to provide decreasing hypoglycemia, weight gain, blood pressure, and GV is necessarily needed.

Dapagliflozin, a member of sodium-glucose cotransporter-2 (SGLT2) inhibitors, was recently approved for the treatment of T2D. Dapagliflozin brings to improvement in glucose control mainly by targeting the pathophysiologic increase in renal glucose reabsorption, leading to increased glucose excretion in the urine [12]. The pleiotropic effects beyond glucose-lowering in T2D associated with macro- and microvascular complications have been proven [13]. Of importance, previous studies have demonstrated that patients treated with dapagliflozin have significant improvements in glycemic control [14-19], with no increase 
in hypoglycemia [20]. We have previously reported that newly diagnosed T2D patients treated with dapagliflozin alone for 24 weeks show improvement in GV and a reduction in oxidative stress, compared to those receiving placebo treatment [21]. Therefore, dapagliflozin should be considered as a good candidate adjunct to insulin to avoid dramatic changes of GV in patients with T2D. However, the effects of dapagliflozin adjunct to insulin on $\mathrm{GV}$ in $\mathrm{T} 2 \mathrm{D}$ patients remain elucidated.

Therefore, we aimed to observe whether dapagliflozin adjunct to insulin therapy further improved glycemic control in patients with newly diagnosed T2D compared to insulin therapy alone.

\section{Methods and Materials}

This was a single-centre, randomized, controlled, openlabeled trial performed at the Department of Endocrinology, Nanjing First Hospital, Nanjing Medical University, between July 2018 and March 2019. The inclusion criteria were as follows: (1) patients aged between 18 and 75 years, (2) $\mathrm{HbA}_{1 \mathrm{c}}$ $\geq 9.0 \%$ at screening, and (3) stabilized weight for at least 12 weeks. Patients were excluded from the analysis if they had acute diabetic complications, chronic liver disease (ALT $>2$ times upper limit of the reference value), kidney disease (Estimated Glomerular Filtration Rate (eGFR) $<60$ $\mathrm{ml} /\left(\min * 1.73 \mathrm{~m}^{2}\right)$, eGFR calculated by the MDRD Study Equation), an infection condition, or cardiovascular disease.

All procedures followed were in accordance with the ethical standards of Nanjing First Hospital and with the Helsinki Declaration of 1964, as revised in 2013. Informed consent was obtained from all the patients for inclusion in the study.

After baseline parameters were assessed, patients were randomly assigned in a $1: 1$ ratio to receive $10 \mathrm{mg}$ dapagliflozin (Bristol-Myers Squibb, Lawrenceville, NJ), plus continuous subcutaneous insulin infusion (CSII), referred to as the DAPA group, or CSII therapy alone, CSII group, for 5 weeks ( 1 week in hospital and another 4 weeks at home). The total daily insulin (Aspart, Novo Nordisk, Bagsværd, Denmark) dose was $0.5 \mathrm{IU} / \mathrm{kg}$ which was given in two injection modes: half the total daily dose was equally given as boluses with three meals, while the remaining insulin was given as a basal dose. Investigators titrated insulin doses on an individualpatient basis using the titration algorithm, as described previously [22]. Scheduled visits occurred once a week for the 5 weeks. The treatment protocol remained unchanged during the study period (Figure 1). Patients were recharged if they achieved $80 \%$ fasting blood glucose levels within 4.4$7.0 \mathrm{mmol} / \mathrm{L}$ and postprandial glucose within 4.4$10.0 \mathrm{mmol} / \mathrm{L}$ for 3 consecutive days. Patients were instructed to maintain their usual diet and exercise at home, and investigations titrated insulin doses according to -2 days of selfmonitoring blood glucose (before and $2 \mathrm{~h}$ after three meals each day and before bedtime) on each scheduled weekly visit. Investigators titrated insulin doses on an individualpatient basis using the titration algorithm: if the fasting blood glucose level was less than $4.4 \mathrm{mmol} / \mathrm{L}$, the basal insulin dose was reduced by 2 units; if the fasting blood glucose level was within $4.4-7.0 \mathrm{mmol} / \mathrm{L}$, the basal insulin dose was unchanged; if the fasting blood glucose level was within $6.2-7.8,7.9-10.0$, or $>10.0 \mathrm{mmol} / \mathrm{L}$, the basal insulin dose was increased subsequently by 2,4 , or 6 units, respectively; if the postprandial blood glucose level was less than $4.4 \mathrm{mmol} / \mathrm{L}$, the bolus insulin dose was reduced by 2 units; if the postprandial blood glucose level was within 4.4$10.0 \mathrm{mmol} / \mathrm{L}$, the bolus insulin dose was unchanged; if the postprandial blood glucose level was $>10.0 \mathrm{mmol} / \mathrm{L}$, the bolus insulin dose was increased subsequently by 2 units, respectively, as described previously $[23,24]$.

All recruited patients were subjected to two-time continuous glucose monitoring (CGM) (Sof-sensor, CGMS-Gold, Medtronic Incorporated, Northridge, USA) for days -3-0 and 36-39 of the study. The CGM sensor was subcutaneously embedded at day -3 and day 36 at 16:00-17:00 PM. During the two-time 3-day CGM periods, the investigator nurses checked the sensor and entered at least 4 calibration readings each day. The sensors were removed, and the CGM data were saved by the investigator, as described previously $[22,25,26]$. All patients were served three meals at 0700,1100 , and 1700, consisting of a total daily caloric intake of $25 \mathrm{kcal} / \mathrm{kg} /$ day. The proportions of carbohydrates, proteins, and fats were $55 \%, 17 \%$, and $28 \%$, respectively. In addition, standard meal tests were performed on days $-3,7$, and 35. Serum samples were collected at 0,30 , and $120 \mathrm{~min}$ after meals for determination of glucose, insulin, and C-peptide concentrations.

The $24 \mathrm{~h}$ mean glucose (MG), the standard deviation of the MG (SDMG), the mean amplitude of glycemic excursions (MAGEs), the incremental area under curve (AUC) of blood glucose above $10.0 \mathrm{mmol} / \mathrm{L}$, the incremental area over the curve (AOC) less than $3.9 \mathrm{mmol} / \mathrm{L}$, and the time in target range (TIR) were recorded and calculated, as previously described $[22,26]$. Plasma insulin levels were determined using an insulin radioimmunoassay kit (Beijing Technology Company, Beijing, China). $\mathrm{HbA}_{1 \mathrm{c}}$ was measured by a DiaSTAT $\mathrm{HbA}_{1 \mathrm{c}}$ analyzer (Bio-Rad, Hercules, CA, USA). C-peptide and glucose concentrations were measured centrally at the central laboratory in Nanjing First Hospital, Nanjing Medical University. Beta cell function was assessed by the homoeostasis model assessment $\mathrm{B}$ (HOMA-B), and insulin sensitivity was indicated by HOMA-IR [27, 28].

The primary endpoint was the difference in MAGE between the two groups at the endpoint. Secondary outcomes were the differences in precise insulin doses, hourly MG, $24 \mathrm{~h}$ MG, SDMG, CV, incremental AUC of hyperglycemia, incremental AOC of hypoglycemia, and weight change from baseline to the completion of treatments. Beta cell function was assessed by HOMA-B, and the insulin sensitivity (HOMA-IR) was also analyzed.

This study was registered with ClinicalTrials.gov (NCT04120623).

2.1. Statistical Analysis. Data were analyzed using the SPSS PASW Statistics 18 Package. The Shapiro-Wilk test was used to assess the distribution of data. Normally distributed and continuous variables were presented as mean \pm standard 


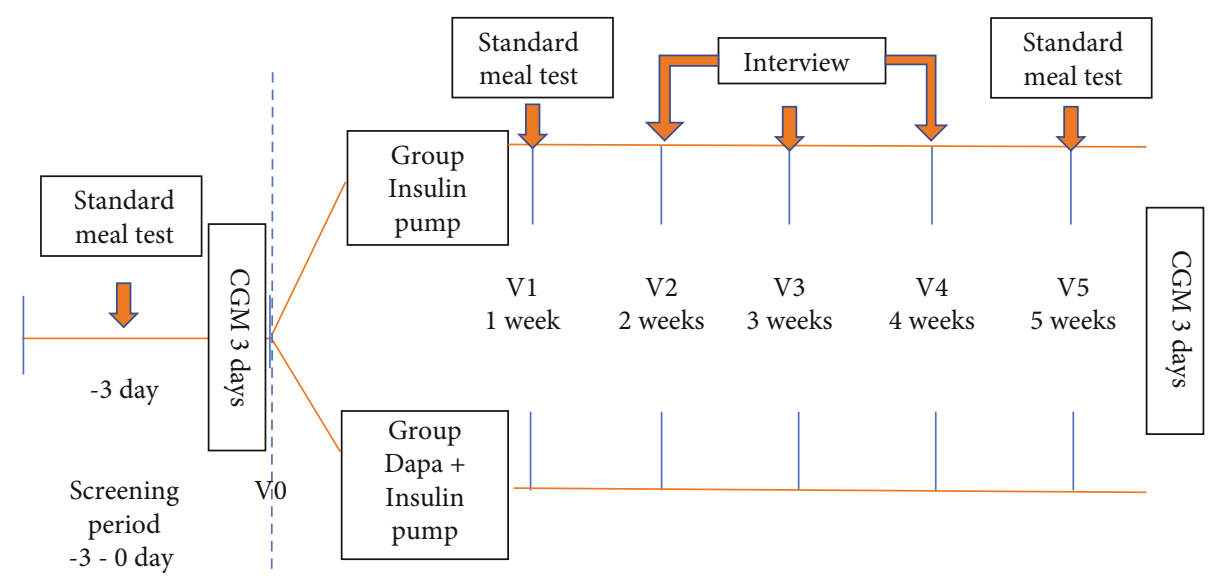

FIgURe 1: Study flow chart.

deviation(SD), while nonnormally distributed variables were presented as median (interquartile range). An independent $t$-test and Wilcoxon test were used in the comparisons between groups. The mixed ANOVA model $(2 \times 2)$ test was used to compare differences between groups. A Bonferroni correction was also performed. $P$ values were two-tailed with a significance level of $5 \%$.

\section{Results}

3.1. Baseline Characteristics. A total of 66 newly diagnosed T2D patients who met the inclusion criteria were admitted to the study, 34 in the DAPA group and 32 in the CSII group. All recruited subjects completed the study (Table 1). Importantly, there were no significant differences in the demographic characteristics of the patients between the two groups.

3.2. Glycemic Variations. Subjects in the DAPA group exhibited significant decreases in the MAGE, incremental AUC hyperglycemia, incremental AOC of hypoglycemia, and TIR at the endpoint of the study compared to those in the CSII group (Table 2). We also observed a trend towards a reduction in the SDMG, $24 \mathrm{~h} \mathrm{MG}$, and the $\mathrm{CV}$ in patients in the DAPA group compared to the CSII group; however, these were not significant. We calculated readings delivered from CGM every $5 \mathrm{~min}$, and we observed that patients in the DAPA group had a significant decrease in hourly mean glucose at 0700, 0800, and 0900 compared to those in the CSII group (Figure 2). As patients had their meals at 0700, 1100 , and 1700, we calculated the incremental AUC of glucose before and after each meal. Our data showed that patients in the DAPA group had a statistically significant decrease in AUC $1 \mathrm{~h}$ before breakfast and $1 \mathrm{~h}, 2 \mathrm{~h}$, and $3 \mathrm{~h}$ after breakfast (Table 3). Hypoglycemia is an important concern regarding insulin therapy in patients with T2D. Therefore, we identified hypoglycemia episodes from CGM readings and found that none of the patients in the DAPA group experienced hypoglycemia. However, a total of 4 patients had hypoglycemic episodes in the CSII group $(P=0.05)$ (Table 2).
3.3. Beta Cell Function and Insulin Sensitivity. To determine the effect of dapagliflozin therapy on beta cell function and insulin sensitivity in patients with newly diagnosed T2D, we compared HOMA-B and HOMA-IR at 1 week of treatment and the endpoint after treatment completion between the two groups. Our data showed that, as expected, patients in the DAPA group had lower HOMA-IR values than those in the CSII group at 1 week of this study, and this improvement remained at the endpoint $(P<0.05$, for both). Interestingly, patients in the DAPA group did not show significant improvement in HOMA-B at 1 week $(P=0.06)$, but achieved a significantly increase in HOMA-B values after 5 weeks of dapagliflozin adjunct to insulin therapy compared to those in the CSII group $(P=0.01)$ (Table 4$)$.

3.4. Insulin and Weight Gain. Patients in the DAPA group reached glycemic goals in a shorter amount of time than those in the CSII group $(3.07 \pm 0.92$ days vs. $3.89 \pm 1.14$ days, $P<0.01)$. The daily total insulin dose required by subjects to maintain euglycemic control in the DAPA group was significantly lower than that of the CSII group after 5 weeks $(0.23 \pm 0.09 \mathrm{U} / \mathrm{kg}$ vs. $0.30 \pm 0.11 \mathrm{U} / \mathrm{kg}, P=0.02)$. We next compared the bolus insulin and basal insulin doses required in the patients between the two groups. We observed that patients in the DAPA group required significantly lower bolus insulin doses compared to those in the CSII group $(P=0.05)$, and our data also indicated that dapagliflozin treatment exhibited a significantly lower basal insulin doses after 5 weeks $(P=0.03)$ (Table 5$)$. In addition, we observed that patients receiving dapagliflozin treatment had a significant reduction in body weight compared to those receiving insulin therapy $(P<0.01)$ (Table 1$)$.

In addition, we also observed a remission of $52.9 \%$ and $46.9 \%$ in patients with CSII+DAPA and CSII alone therapy after one-year follow-up, respectively.

3.5. Safety and Tolerance. We compared the experienced side effects during the study period. One patient experienced a moderate urinary tract infection during dapagliflozin therapy at 2 weeks, but he continued to complete the study. Other patients tolerated the dapagliflozin or insulin therapy well, without any adverse reactions recorded. 
TABLE 1: Baseline characteristics of patients.

\begin{tabular}{|c|c|c|c|c|c|c|c|c|}
\hline \multirow[b]{2}{*}{ Parameter } & \multicolumn{3}{|c|}{ Before therapy } & \multicolumn{5}{|c|}{ After therapy $(5 \mathrm{w})$} \\
\hline & $\mathrm{DAPA}+\mathrm{CSII}(n=34)$ & CSII $(n=32)$ & $P$ value & $\mathrm{DAPA}+\mathrm{CSII}(n=34)$ & CSII $(n=32)$ & $\begin{array}{c}\triangle \mathrm{DAPA} \\
+\mathrm{CSII}\end{array}$ & $\triangle \mathrm{CSII}$ & $P$ value \\
\hline Gender $(\mathrm{M} / \mathrm{F})$ & $34(22 / 12)$ & $32(26 / 6)$ & 0.13 & 1 & 1 & 1 & 1 & 1 \\
\hline Age (years) & $50.0 \pm 10.4$ & $46.3 \pm 10.1$ & 0.15 & l & l & l & l & l \\
\hline Weight (kg) & $72.6 \pm 9.9$ & $69.5 \pm 12.2$ & 0.26 & $70.0 \pm 9.7$ & $68.2 \pm 12.1$ & $-2.6 \pm 0.7$ & $-1.3 \pm 0.8$ & $<0.01^{* *}$ \\
\hline BMI $\left(\mathrm{kg} / \mathrm{m}^{2}\right)$ & $25.6 \pm 3.0$ & $24.1 \pm 3.4$ & 0.08 & $24.7 \pm 3.0$ & $23.7 \pm 3.4$ & $-0.9 \pm 0.2$ & $-0.45 \pm 0.3$ & $<0.01^{* *}$ \\
\hline $\mathrm{SBP}(\mathrm{mmHg})$ & $125.9 \pm 13.3$ & $125.4 \pm 12.0$ & 0.89 & $120.5 \pm 8.6$ & $120.5 \pm 10.6$ & $-5.4 \pm 13.0$ & $-4.9 \pm 14.1$ & 0.90 \\
\hline DBP (mmHg) & $81.6 \pm 7.5$ & $81.7 \pm 4.8$ & 0.97 & $78.7 \pm 5.9$ & $80.9 \pm 4.6$ & $-2.6 \pm 5.1$ & $-0.7 \pm 4.8$ & 0.09 \\
\hline $\mathrm{Na}^{+}(\mathrm{mmol} / \mathrm{L})$ & $142.6 \pm 2.4$ & $142.8 \pm 2.8$ & 0.74 & $143.9 \pm 2.2$ & $143.0 \pm 2.5$ & $-1.5 \pm 3.0$ & $-0.2 \pm 3.3$ & 0.14 \\
\hline $\mathrm{HbA}_{1 \mathrm{c}}(\%)$ & $10.2 \pm 1.77$ & $10.5 \pm 1.6$ & 0.52 & $7.8 \pm 0.9$ & $8.1 \pm 1.0$ & $-2.5 \pm 1.2$ & $-2.4 \pm 1.2$ & 0.93 \\
\hline $\mathrm{FBG}(\mathrm{mmol} / \mathrm{L})$ & $11.9 \pm 2.8$ & $11.3 \pm 2.0$ & 0.30 & $6.9 \pm 1.4$ & $7.5 \pm 2.0$ & $-5.0 \pm 3.1$ & $-3.8 \pm 2.3$ & 0.08 \\
\hline
\end{tabular}

BMI: body mass index; SBP: systolic blood pressure; DBP: diastolic blood pressure; FBG: fasting blood glucose; $\mathrm{HbA}_{1 \mathrm{c}}$ : hemoglobin A1c. Data were presented as means $\pm \mathrm{SD} ;{ }^{*} P<0.05 ;{ }^{* *} P<0.01 . \triangle$ : before therapy-after therapy $(5 \mathrm{w})$.

TABLE 2: Blood glucose variability in the recruited subjects.

\begin{tabular}{|c|c|c|c|c|c|c|}
\hline \multirow{2}{*}{ Parameter } & \multicolumn{2}{|c|}{ Before therapy } & \multirow{2}{*}{$P$ value } & \multicolumn{2}{|c|}{ After therapy $(5 \mathrm{w})$} & \multirow{2}{*}{$P$ value } \\
\hline & DAPA+CSII & CSII & & $\mathrm{DAPA}+\mathrm{CSII}$ & CSII & \\
\hline MAGE & $6.25 \pm 2.55$ & $5.97 \pm 2.47$ & 0.68 & $2.34 \pm 1.10$ & $3.46 \pm 2.33$ & $0.03^{*}$ \\
\hline MBG & $12.15 \pm 2.37$ & $12.28 \pm 2.14$ & 0.83 & $6.60 \pm 0.98$ & $7.19 \pm 1.65$ & 0.10 \\
\hline SD & $2.52 \pm 0.99$ & $2.50 \pm 0.90$ & 0.94 & $1.09 \pm 0.63$ & $1.42 \pm 0.70$ & 0.07 \\
\hline CV\% & $20.54 \pm 6.48$ & $20.20 \pm 5.95$ & 0.84 & $16.21 \pm 7.90$ & $19.27 \pm 7.79$ & 0.14 \\
\hline AUC $>10 \mathrm{mmol} / \mathrm{L}$ & $2.05(1.19,4.20)$ & $2.48(0.90,3.93)$ & 0.89 & $0(0,0)$ & $0.02(0,0.40)$ & $0.01^{*}$ \\
\hline $\mathrm{AOC}<3.9 \mathrm{mmol} / \mathrm{L}$ & $0(0,0)$ & $0(0,0)$ & 0.33 & $0(0,0)$ & $0(0,0)$ & $0.03^{*}$ \\
\hline Hypoglycemia $(n)$ & 0 & 0 & l & 0 & 4 & 0.05 \\
\hline TIR & $26.35 \pm 26.64$ & $31.95 \pm 25.17$ & 0.42 & $96.52 \pm 8.28$ & $85.09 \pm 19.08$ & $0.01^{*}$ \\
\hline
\end{tabular}

MAGE: the mean amplitude of glycemic excursion; MBG: $24 \mathrm{~h}$ mean blood glucose; SD: standard deviation of mean glucose; CV\%: the coefficient of variation; AUC $>10 \mathrm{mmol} / \mathrm{L}$ : the incremental area under the curve (AUC) of a glucose level $>10.0 \mathrm{mmol} / \mathrm{L} ; \mathrm{AOC}<3.9 \mathrm{mmol} / \mathrm{L}$ : the incremental area over the curve $(\mathrm{AOC})$ of a glucose level $<3.9 \mathrm{mmol} / \mathrm{L}$; TIR: the time in target range $(3.9-10.0 \mathrm{mmol} / \mathrm{L})$. Data were presented as means $\pm \mathrm{SD}$ or IQR. ${ }^{*} P<0.05 ;{ }^{* *} P<0.01$.

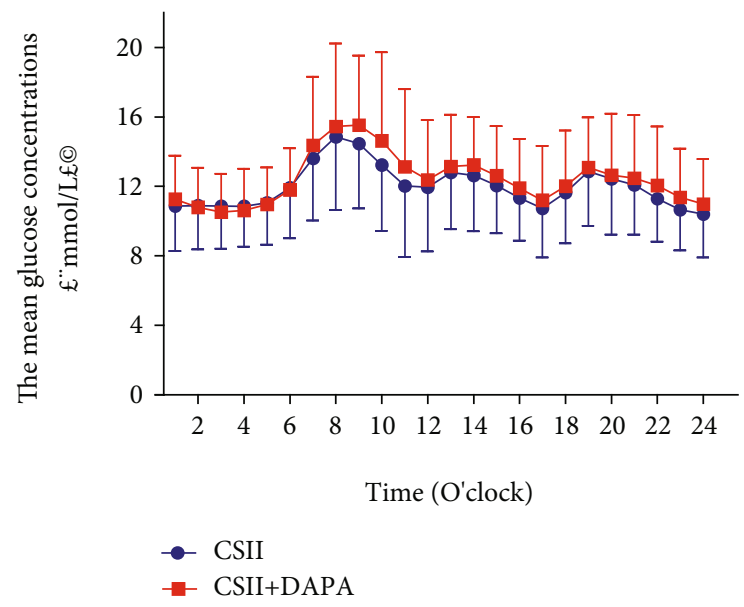

(a)

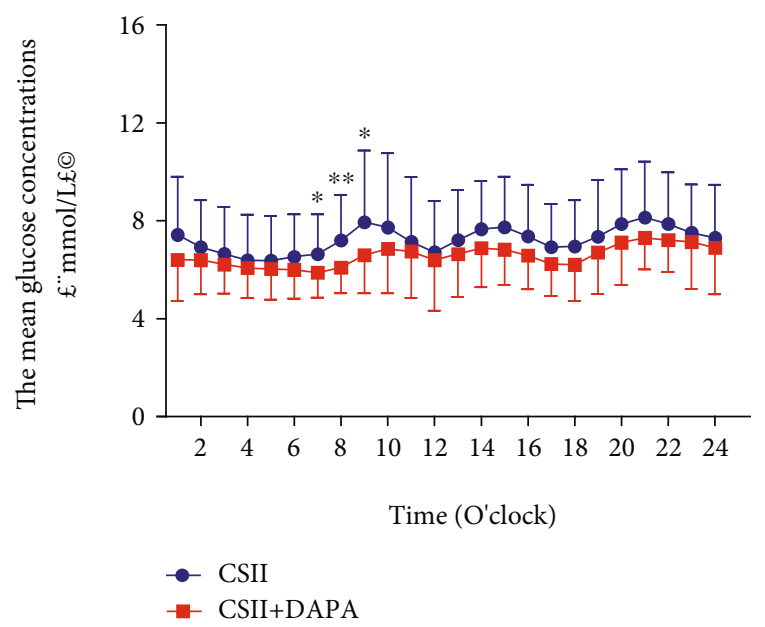

(b)

FIGURE 2: Hourly glucose concentrations between the two groups: (a) before therapy and (b) after therapy. Red line: CSII+DAPA group and blue line: CSII group; ${ }^{*} P<0.05 ;{ }^{* *} P<0.01$. 
TABLE 3: The incremental AUC of glucose values before and after each meal.

\begin{tabular}{|c|c|c|c|c|c|c|}
\hline \multirow{2}{*}{ Parameter } & \multicolumn{2}{|c|}{ Before therapy } & \multirow{2}{*}{$P$ value } & \multicolumn{2}{|c|}{ After therapy } & \multirow{2}{*}{$P$ value } \\
\hline & DAPA+CSII & CSII & & $\mathrm{DAPA}+\mathrm{CSII}$ & CSII & \\
\hline \multicolumn{7}{|l|}{ Breakfast } \\
\hline AUCb-1 h & $3.04 \pm 0.86$ & $2.86 \pm 0.76$ & 0.42 & $1.22 \pm 0.21$ & $1.38 \pm 0.34$ & $0.03^{*}$ \\
\hline AUCa-1 h & $3.22 \pm 0.99$ & $3.10 \pm 0.87$ & 0.62 & $1.27 \pm 0.23$ & $1.51 \pm 0.40$ & $0.01^{*}$ \\
\hline AUCa-2 h & $6.45 \pm 1.73$ & $6.10 \pm 1.59$ & 0.42 & $2.64 \pm 0.52$ & $3.17 \pm 0.98$ & $0.01^{*}$ \\
\hline AUCa-3 h & $9.49 \pm 2.67$ & $8.85 \pm 2.20$ & 0.32 & $4.08 \pm 0.78$ & $4.77 \pm 1.54$ & $0.03^{*}$ \\
\hline AUCa-4h & $12.21 \pm 3.51$ & $11.34 \pm 2.83$ & 0.31 & $5.48 \pm 1.01$ & $6.25 \pm 1.98$ & 0.06 \\
\hline \multicolumn{7}{|l|}{ Lunch } \\
\hline AUCb-1 h & $2.72 \pm 0.92$ & $2.50 \pm 0.85$ & 0.34 & $1.40 \pm 0.40$ & $1.48 \pm 0.55$ & 0.53 \\
\hline AUCa-1 h & $2.58 \pm 0.70$ & $2.50 \pm 0.76$ & 0.67 & $1.33 \pm 0.43$ & $1.40 \pm 0.43$ & 0.58 \\
\hline AUCa-2 h & $5.32 \pm 1.26$ & $5.17 \pm 1.36$ & 0.65 & $2.72 \pm 0.76$ & $2.91 \pm 0.83$ & 0.37 \\
\hline AUCa-3 h & $8.08 \pm 1.74$ & $7.80 \pm 1.93$ & 0.56 & $4.15 \pm 1.00$ & $4.51 \pm 1.13$ & 0.21 \\
\hline AUCa-4h & $10.70 \pm 2.26$ & $10.30 \pm 2.41$ & 0.52 & $5.58 \pm 1.19$ & $6.12 \pm 1.48$ & 0.13 \\
\hline \multicolumn{7}{|l|}{ Dinner } \\
\hline AUCb-1 h & $2.33 \pm 0.65$ & $2.23 \pm 0.59$ & 0.54 & $1.30 \pm 0.27$ & $1.44 \pm 0.36$ & 0.10 \\
\hline AUCa-1 h & $2.52 \pm 0.67$ & $2.45 \pm 0.61$ & 0.66 & $1.28 \pm 0.34$ & $1.45 \pm 0.40$ & 0.08 \\
\hline AUCa-2 h & $5.24 \pm 1.16$ & $5.12 \pm 1.21$ & 0.70 & $2.64 \pm 0.73$ & $3.00 \pm 0.84$ & 0.09 \\
\hline AUCa-3 h & $7.87 \pm 1.82$ & $7.70 \pm 1.79$ & 0.72 & $4.08 \pm 1.13$ & $4.64 \pm 1.26$ & 0.08 \\
\hline AUCa-4h & $10.46 \pm 2.44$ & $10.21 \pm 2.28$ & 0.69 & $5.69 \pm 1.13$ & $6.34 \pm 1.62$ & 0.08 \\
\hline
\end{tabular}

AUC: area under the curve (day $* \mathrm{mmol} / \mathrm{L})$; AUCb: area under the curve before meal (day $* \mathrm{mmol} / \mathrm{L}) ; \mathrm{AUCa}$ area under the curve after meal (day $* \mathrm{mmol} / \mathrm{L})$; ${ }^{*} P<0.05$.

\section{Discussion}

We conducted a prospective study on patients with newly diagnosed T2D and demonstrated that dapagliflozin addon intensive insulin therapy led to a significant improvement in GV. We also observed that patients receiving dapagliflozin with insulin had improved insulin sensitivity and beta cell function.

Studies have demonstrated that dapagliflozin can be well tolerated in patients with T2D for more than 2 years [20, 29], accompanying with blood pressure reduction and cardiovascular and renal benefits 13. Importantly, clinical trials have suggested the potential benefits of SGLT2 inhibitors as an adjunctive treatment for type 1 diabetes (T1D) [30], especially in combination with insulin therapy to improve glycemic control in patients with inadequately controlled TID [31]. The DEPICT-2 Study demonstrated that dapagliflozin at $5 \mathrm{mg}$ or $10 \mathrm{mg}$ add-on insulin therapy was safe and well tolerated and exhibited a potential benefit in improving glycemic control and hypoglycemia in T1D [32]. In our study, type 2 diabetic patients received dapagliflozin $(10 \mathrm{mg})$ adjunctive to insulin therapy were well tolerated for 5 weeks.

As expected, our CGM data showed that patients treated with dapagliflozin exhibited significantly improved GV, such as MAGE, incremental AUC of hyperglycemia, and TIR. Importantly, patients who received dapagliflozin had a statistically significant decrease in the incremental AOC of hypoglycemia. Our data were consistent with studies reporting that dapagliflozin had the ability to improve glycemic control [14-19], with no increase in hypoglycemia [20]. Furthermore, we observed that patients who received dapagliflozin in combination with insulin therapy showed a reduction in $24 \mathrm{~h} \mathrm{MBG}$, which was consistent with our previous study showing that subjects with dapagliflozin therapy had significantly reduced $24 \mathrm{~h}$ MBG compared with placebo after 24 weeks of treatment [21]. Dapagliflozin reduces hyperglycemia, body weight, and systolic blood pressure $[17,33]$ and increases atrial natriuretic peptide levels [34], which may contribute to the decrease in the incidence of cardiovascular disease (CVD). The reduction we showed in glycemic variations may contribute to the reduction of decrease in CVD risk, as acute glucose variations during postprandial periods had a potential role in oxidative stress in patients with T2D [35]. Furthermore, a large GV induced the overproduction of peroxynitrite and nitrotyrosine, which impaired endothelial cell functions $[35,36]$.

Dapagliflozin add-on to insulin therapy leads to a reduction in insulin dose and weight loss, without any increases in hypoglycemia in T1D [37]. In this study, we observed that dapagliflozin as an adjunct to insulin significantly reduced basal and bolus insulin doses in T2D after 5-week treatment, with no weight changes and hypoglycemia. We also analyzed $\beta$-cell function and insulin sensitivity in patients between groups. Interestingly, subjects receiving dapagliflozin addon therapy had statistically improved in insulin sensitivity and beta cell function. However, our data indicated that patients achieved the recovery of beta cell function was seen at the endpoint (5-week treatment), which may be the 


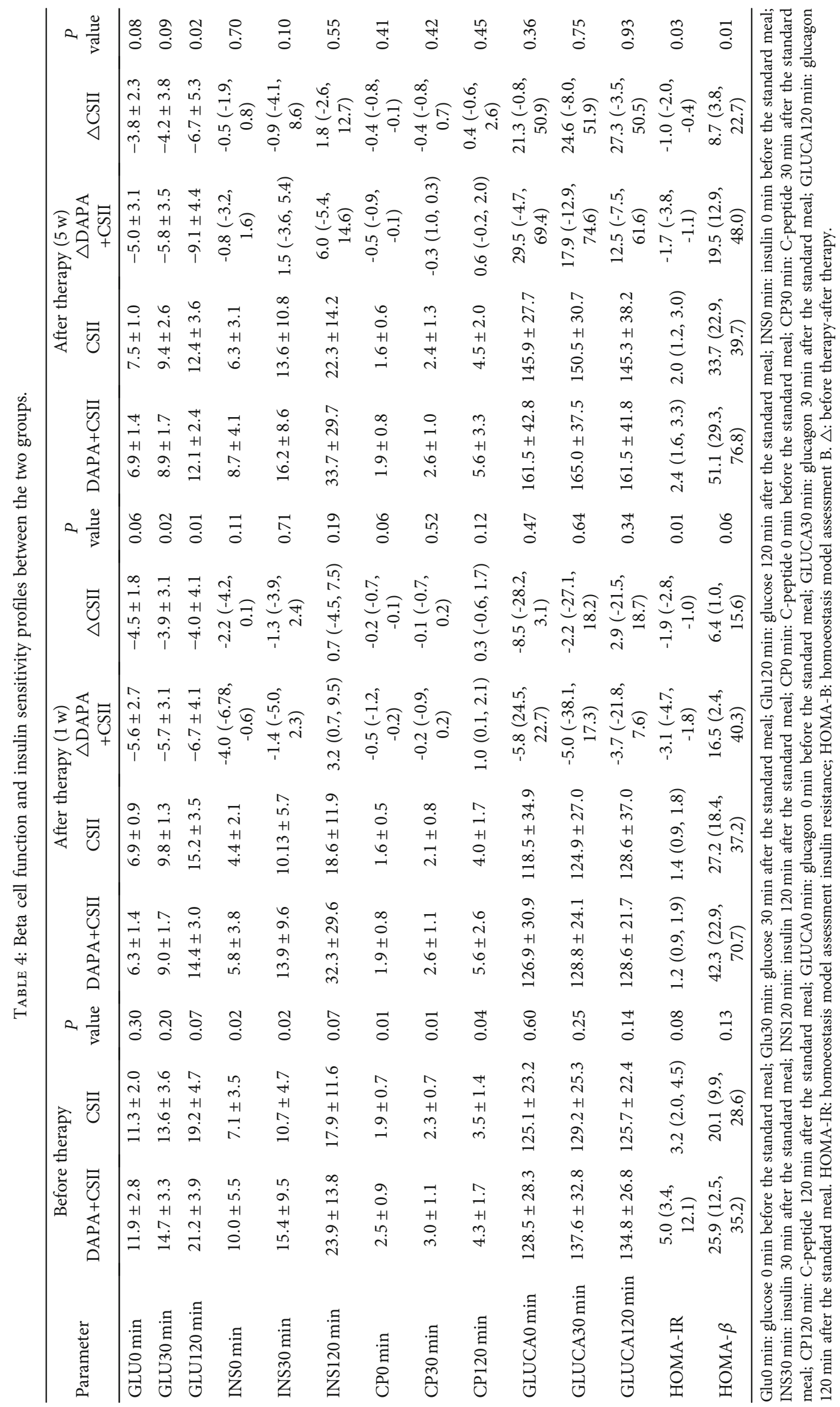


TABLE 5: Insulin doses required by patients in the two groups.

\begin{tabular}{|c|c|c|c|c|c|c|}
\hline \multirow{2}{*}{ Parameter } & \multicolumn{2}{|c|}{ Therapy for 1 week } & \multirow{2}{*}{$P$ value } & \multicolumn{2}{|c|}{ Therapy for 5 weeks } & \multirow{2}{*}{$P$ value } \\
\hline & $\mathrm{DAPA}+\mathrm{CSII}$ & CSII & & $\mathrm{DAPA}+\mathrm{CSII}$ & CSII & \\
\hline Total (U/kg) & $0.36 \pm 0.12$ & $0.39 \pm 0.12$ & 0.45 & $0.23 \pm 0.09$ & $0.30 \pm 0.11$ & 0.02 \\
\hline Basal (U/kg) & $0.20 \pm 0.07$ & $0.21 \pm 0.06$ & 0.49 & $0.12 \pm 0.06$ & $0.16 \pm 0.06$ & 0.03 \\
\hline Total bolus (U/kg) & $0.17 \pm 0.07$ & $0.18 \pm 0.07$ & 0.50 & $0.11 \pm 0.05$ & $0.14 \pm 0.06$ & 0.03 \\
\hline Bolus (B) & $0.06 \pm 0.03$ & $0.06 \pm 0.03$ & 0.56 & $0.04 \pm 0.02$ & $0.05 \pm 0.02$ & 0.05 \\
\hline Bolus (L) & $0.06 \pm 0.02$ & $0.06 \pm 0.02$ & 0.60 & $0.08 \pm 0.02$ & $0.05 \pm 0.02$ & 0.03 \\
\hline Bolus (D) & $0.06 \pm 0.02$ & $0.06 \pm 0.02$ & 0.44 & $0.03 \pm 0.01$ & $0.04 \pm .022$ & 0.05 \\
\hline
\end{tabular}

Bolus (B): bolus breakfast; bolus (L): bolus lunch; bolus (D): bolus dinner.

reason of SGLT2 inhibitors have the potential ability to preserve beta cell mass in diabetic mouse model [38].

An array of metrics delivered from CGM could be used to interpret the GV in T1D and T2D [39-41]. Our CGM data showed that patients receiving dapagliflozin in combination with insulin therapy showed a significant improvement in MAGE, incremental AUC, and TIR. In addition, we also observed that subjects in the DAPA group had a significant decrease in the incremental AUC at $3 \mathrm{~h}$ postbreakfast compared to the CSII group. Therefore, it would be more logical to hypothesis that patients with higher $\mathrm{HbA}_{1 \mathrm{c}}$ values may harvest more benefit from dapagliflozin adjunct to insulin therapy for the improvement in incremental AUC after breakfast. However, our data could not address the underlying mechanisms of this decrease in incremental AUC after breakfast. We could infer that decreased incremental AUC after breakfast might be the reason for nearly half of the newly diagnosed T2D patients with only abnormal postprandial glucose concentrations [42]. Furthermore, isolated postprandial hyperglycemia is more prominent in Chinese patients compared to Western patients [42, 43]. This was strengthened by our study reporting that newly diagnosed T2D patients with higher $\mathrm{HbA}_{1 \mathrm{c}}$ values had larger GV and higher peak glucose concentrations after breakfast. Limitations of this study should be addressed; in particular, the study population was relatively small, and the observation time was relatively short. However, the strength and novelty of this study should also be addressed, especially this study was performed under very controlled conditions in the hospital setting (which is quite unique) and with the use of CGM, thus providing valuable and reliable data on the effect of dapagliflozin on $\mathrm{GV}$ in patients managed with state-ofthe-art CSII.

In conclusion, our data indicate that dapagliflozin adjunct to insulin is a safe and effective therapy to improve glycemic variations, insulin sensitivity, and weight loss in newly diagnosed T2D patients.

\section{Data Availability}

The datasets generated and/or analyzed during the current study are not publicly available due to some reasons but are available from the corresponding author on reasonable request.

\section{Conflicts of Interest}

All authors declare that there is no conflict of interest.

\section{Authors' Contributions}

JH. M. and FF. L. contributed to the conception and design of the study. LL. J. and P. Z. contributed to the conduct/data collection. BL. L. and LL. J. contributed to data analysis. FF. L. contributed to manuscript writing. JH. M. and L.Y. contributed to the final approval of the manuscript. Lan-lan Jiang, Peng Zhang, and Bing-li Liu contributed equally to this work.

\section{Acknowledgments}

This research was funded by the National Key R\&D Program of China (2018YFC1314100), the Nanjing Municipal Science and Technology Project (No. 201803011), and the Science and Technology Development Foundation of Nanjing Medical University (Nos. 2017NJMUZD108 and 2017NJMU128).

\section{References}

[1] J. Tuomilehto, J. Lindström, J. G. Eriksson et al., "Prevention of type 2 diabetes mellitus by changes in lifestyle among subjects with impaired glucose tolerance," The New England Journal of Medicine, vol. 344, no. 18, pp. 1343-1350, 2001.

[2] L. J. Orozco, A. M. Buchleitner, G. Gimenez-Perez et al., "Exercise or exercise and diet for preventing type 2 diabetes mellitus," The Cochrane Database of Systematic Reviews, vol. 3, article CD003054, 2008.

[3] S. Min Lee, J. Baik, D. Nguyen et al., "Kcne2deletion impairs insulin secretion and causes type 2 diabetes mellitus," FASEB Journal : Official Publication of the Federation of American Societies for Experimental Biology, vol. 31, no. 6, pp. 26742685, 2017.

[4] L. A. Donnelly, A. D. Morris, B. M. Frier et al., "Frequency and predictors of hypoglycaemia in type 1 and insulin-treated type 2 diabetes: a population-based study," Diabetic medicine : a journal of the British Diabetic Association, vol. 22, no. 6, pp. 749-755, 2005.

[5] D. M. Nathan and for the DCCT/EDIC Research Group, "The diabetes control and complications trial/epidemiology of diabetes interventions and complications study at 30 years: overview," Diabetes Care, vol. 37, no. 1, pp. 9-16, 2014. 
[6] UK Hypoglycaemia Study Group, "Risk of hypoglycaemia in types 1 and 2 diabetes: effects of treatment modalities and their duration," Diabetologia, vol. 50, no. 6, pp. 1140-1147, 2007.

[7] F. F. Li, Y. Zhang, W. L. Zhang et al., "Male patients with longstanding type 2 diabetes have a higher incidence of hypoglycemia compared with female patients," Diabetes Therapy, vol. 9, no. 5, pp. 1969-1977, 2018.

[8] J. Q. Purnell, J. E. Hokanson, S. M. Marcovina, M. W. Steffes, P. A. Cleary, and J. D. Brunzell, "Effect of excessive weight gain with intensive therapy of type 1 diabetes on lipid levels and blood Pressure," JAMA, vol. 280, no. 2, pp. 140-146, 1998.

[9] D. Russell-Jones and R. Khan, "Insulin-associated weight gain in diabetes - causes, effects and coping strategies," Diabetes, Obesity \& Metabolism, vol. 9, no. 6, pp. 799-812, 2007.

[10] A. Wallia and M. E. Molitch, "Insulin therapy for type 2 diabetes mellitus," JAMA, vol. 311, no. 22, pp. 2315-2325, 2014.

[11] C. S. Frandsen, T. F. Dejgaard, and S. Madsbad, "Non-insulin drugs to treat hyperglycaemia in type 1 diabetes mellitus," The Lancet Diabetes \& Endocrinology, vol. 4, no. 9, pp. 766-780, 2016.

[12] D. Vasilakou, T. Karagiannis, E. Athanasiadou et al., "Sodiumglucose cotransporter 2 inhibitors for type 2 diabetes: a systematic review and meta-analysis," Annals of Internal Medicine, vol. 159, no. 4, pp. 262-274, 2013.

[13] H. J. Heerspink, B. A. Perkins, D. H. Fitchett, M. Husain, and D. Z. Cherney, "Sodium glucose cotransporter 2 inhibitors in the treatment of diabetes mellitus: cardiovascular and kidney effects, potential mechanisms, and clinical applications," Circulation, vol. 134, no. 10, pp. 752-772, 2016.

[14] C. J. Bailey, E. C. Morales Villegas, V. Woo, W. Tang, A. Ptaszynska, and J. F. List, "Efficacy and safety of dapagliflozin monotherapy in people with type 2 diabetes: a randomized double-blind placebo-controlled 102-week trial," Diabetic medicine : a journal of the British Diabetic Association, vol. 32, no. 4, pp. 531-541, 2015.

[15] J. P. Wilding, V. Woo, K. Rohwedder, J. Sugg, S. Parikh, and for the Dapagliflozin 006 Study Group, "Dapagliflozin in patients with type 2 diabetes receiving high doses of insulin: efficacy and safety over 2 years," Diabetes, Obesity \& Metabolism, vol. 16, no. 2, pp. 124-136, 2014.

[16] M. A. Nauck, S. del Prato, J. J. Meier et al., "Dapagliflozin versus glipizide as add-on therapy in patients with type 2 diabetes who have inadequate glycemic control with metformin: a randomized, 52-week, double-blind, active-controlled noninferiority trial," Diabetes Care, vol. 34, no. 9, pp. 2015-2022, 2011.

[17] W. T. Cefalu, L. A. Leiter, T. W. A. de Bruin, I. Gause-Nilsson, J. Sugg, and S. J. Parikh, "Dapagliflozin's effects on glycemia and cardiovascular risk factors in high-risk patients with type 2 diabetes: a 24-week, multicenter, randomized, double-blind, placebo-controlled study with a 28 -week extension," Diabetes Care, vol. 38, no. 7, pp. 1218-1227, 2015.

[18] H. J. Lambers Heerspink, D. de Zeeuw, L. Wie, B. Leslie, and J. List, "Dapagliflozin a glucose-regulating drug with diuretic properties in subjects with type 2 diabetes," Diabetes, Obesity \& Metabolism, vol. 15, no. 9, pp. 853-862, 2013.

[19] K. Strojek, K. H. Yoon, V. Hruba, J. Sugg, A. M. Langkilde, and S. Parikh, "Dapagliflozin added to glimepiride in patients with type 2 diabetes mellitus sustains glycemic control and weight loss over 48 weeks: a randomized, double-blind, parallelgroup, placebo-controlled trial," The Lancet Diabetes \& Endocrinology, vol. 5, no. 1, pp. 267-283, 2014.
[20] L. A. Leiter, W. T. Cefalu, T. W. A. de Bruin et al., "Long-term maintenance of efficacy of dapagliflozin in patients with type 2 diabetes mellitus and cardiovascular disease," Diabetes, Obesity \& Metabolism, vol. 18, no. 8, pp. 766-774, 2016.

[21] F. F. Li, G. Gao, Q. Li et al., "Influence of dapagliflozin on glycemic variations in patients with newly diagnosed type 2 diabetes mellitus," Journal of Diabetes Research, vol. 2016, Article ID 5347262, 6 pages, 2016.

[22] F. F. Li, L. Y. Fu, W. L. Zhang et al., "Blood glucose fluctuations in type 2 diabetes patients treated with multiple daily injections," Journal of Diabetes Research, vol. 2016, Article ID 1028945, 8 pages, 2016.

[23] B. L. Liu, X. M. Liu, Y. F. Ren et al., "Sexual differences in response to mid- or low-premixed insulin analogue in patients with type 2 diabetes," Journal of Diabetes Research, vol. 2020, 7 pages, 2020.

[24] F. F. Li, L. L. Jiang, R. N. Yan et al., "Effects of saxagliptin addon therapy to insulin on blood glycemic fluctuations in patients with type 2 diabetes: a randomized, control, openlabeled trial," Medicine, vol. 95, no. 43, article e5229, 2016.

[25] J. Zhou, H. Li, X. Ran et al., "Reference values for continuous glucose monitoring in Chinese subjects," Diabetes Care, vol. 32, no. 7, pp. 1188-1193, 2009.

[26] F. F. Li, X. H. Xu, L. Y. Fu et al., "Influence of acarbose on plasma glucose fluctuations in insulin-treated patients with type 2 diabetes: a pilot study," International Journal of Endocrinology, vol. 2015, Article ID 903524, 5 pages, 2015.

[27] D. R. Matthews, J. P. Hosker, A. S. Rudenski, B. A. Naylor, D. F. Treacher, and R. C. Turner, "Homeostasis model assessment: insulin resistance and beta-cell function from fasting plasma glucose and insulin concentrations in man," Diabetologia, vol. 28, no. 7, pp. 412-419, 1985.

[28] J. Weng, Y. Li, W. Xu et al., "Effect of intensive insulin therapy on $\beta$-cell function and glycaemic control in patients with newly diagnosed type 2 diabetes: a multicentre randomised parallel-group trial," Lancet, vol. 371, no. 9626, pp. $1753-$ 1760, 2008.

[29] S. del Prato, M. Nauck, S. Durán-Garcia et al., "Long-term glycaemic response and tolerability of dapagliflozin versus a sulphonylurea as add-on therapy to metformin in patients with type 2 diabetes: 4-year data," Diabetes, Obesity \& Metabolism, vol. 17, no. 6, pp. 581-590, 2015.

[30] J. Chen, F. Fan, J. Y. Wang et al., "The efficacy and safety of SGLT2 inhibitors for adjunctive treatment of type 1 diabetes: a systematic review and meta-analysis," Scientific Reports, vol. 7, no. 1, article 44128, 2017.

[31] P. Dandona, C. Mathieu, M. Phillip et al., "Efficacy and safety of dapagliflozin in patients with inadequately controlled type 1 diabetes (DEPICT-1): 24 week results from a multicentre, double-blind, phase 3, randomised controlled trial," The Lancet Diabetes \& Endocrinology, vol. 5, no. 11, pp. 864-876, 2017.

[32] C. Mathieu, P. Dandona, P. Gillard et al., "Efficacy and safety of dapagliflozin in patients with inadequately controlled type 1 diabetes (the DEPICT-2 study): 24-week results from a randomized controlled trial," Diabetes Care, vol. 41, no. 9, pp. 1938-1946, 2018.

[33] L. A. Leiter, W. T. Cefalu, T. W. A. de Bruin, I. Gause-Nilsson, J. Sugg, and S. J. Parikh, "Dapagliflozin added to usual care in individuals with type 2 diabetes mellitus with preexisting cardiovascular disease: a 24-week, multicenter, randomized, double-blind, placebo-controlled study with a 28 -week extension," 
Journal of the American Geriatrics Society, vol. 62, no. 7, pp. 1252-1262, 2014.

[34] Y. Wang, L. Xu, L. Yuan et al., "Sodium-glucose cotransporter-2 inhibitors suppress atrial natriuretic peptide secretion in patients with newly diagnosed type 2 diabetes," Diabetic Medicine, vol. 33, no. 12, pp. 1732-1736, 2016.

[35] L. Monnier, E. Mas, C. Ginet et al., "Activation of oxidative stress by acute glucose fluctuations compared with sustained chronic hyperglycemia in patients with type 2 diabetes," JAMA, vol. 295, no. 14, pp. 1681-1687, 2006.

[36] Y. Hu, W. Liu, R. Huang, and X. Zhang, "Postchallenge plasma glucose excursions, carotid intima-media thickness, and risk factors for atherosclerosis in Chinese population with type 2 diabetes," Atherosclerosis, vol. 210, no. 1, pp. 302-306, 2010.

[37] T. R. Pieber, S. Famulla, J. Eilbracht et al., "Empagliflozin as adjunct to insulin in patients with type 1 diabetes: a 4-week, randomized, placebo-controlled trial (EASE-1)," Diabetes, Obesity \& Metabolism, vol. 17, no. 10, pp. 928-935, 2015.

[38] K. Takahashi, A. Nakamura, H. Miyoshi et al., "Effect of the sodium-glucose cotransporter 2 inhibitor luseogliflozin on pancreatic beta cell mass in $\mathrm{db} / \mathrm{db}$ mice of different ages," Scientific Reports, vol. 8, no. 1, 2018.

[39] C. Fabris, A. Facchinetti, G. Sparacino et al., "Glucose variability indices in type 1 diabetes: parsimonious set of indices revealed by sparse principal component analysis," Diabetes Technology \& Therapeutics, vol. 16, no. 10, pp. 644-652, 2014.

[40] C. Fabris, A. Facchinetti, G. Fico et al., "Parsimonious description of glucose variability in type 2 diabetes by sparse principal component analysis," Journal of Diabetes Science and Technology, vol. 10, no. 1, pp. 119-124, 2016.

[41] D. Rodbard, "New and improved methods to characterize glycemic variability using continuous glucose monitoring," Diabetes Technology \& Therapeutics, vol. 11, no. 9, pp. 551-565, 2009.

[42] W. Yang, J. Lu, J. Weng et al., "Prevalence of diabetes among men and women in China," The New England Journal of Medicine, vol. 362, no. 12, pp. 1090-1101, 2010.

[43] DECODE Study Group on behalf of the European Diabetes Epidemiology Study Group, "Will new diagnostic criteria for diabetes mellitus change phenotype of patients with diabetes? Reanalysis of European epidemiological data," BMJ, vol. 317, no. 7155, pp. 371-375, 1998. 